\title{
Methane storage capabilities of diamond analogues
}

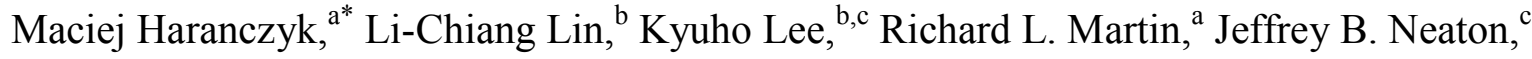 \\ Berend Smit ${ }^{\mathrm{a}, \mathrm{b}, \mathrm{d}}$ \\ ${ }^{a}$ Lawrence Berkeley National Laboratory, One Cyclotron Road, MS 50F-1650, Berkeley, CA 94720-8139, USA \\ ${ }^{b}$ Department of Chemical and Biomolecular Engineering, University of California, Berkeley, CA 94720, USA \\ ${ }^{c}$ Molecular Foundry, Lawrence Berkeley National Laboratory, One Cyclotron Road, MS 50F-1650, Berkeley, CA \\ 94720-8139, USA \\ ${ }^{d}$ Department of Chemistry, University of California, Berkeley, CA 94720, USA \\ *mharanczyk@lbl.gov; Fax +1 510486 5812; Phone +1 510-486-7749
}

\section{Supporting Materials}

\subsection{The band structure of diamond analogues}

The energy band structure of extDIA, extDIA-Si, extDIA-Ge, extDIA-Si-BN and extDIA-BN was calculated using the fully relaxed structures. We used the VASP code with PBE, $400 \mathrm{eV}$ kinetic energy cutoff and $4 \times 4 \times 4 \mathrm{k}$-point sampling for self-consistent charge density calculation. Then non-self-consistent band calculations have been done for the band along the high symmetry lines in the first Brillouin zone. Total $105 \mathrm{k}$-points are sampled for the band plots. The band plots are given in Figure $1 \mathrm{~S}$. We found similar energy gaps $(\sim 4 \mathrm{eV})$ across all analogues. 
Figure 1S. The electronic band structures of extDIA, extDIA-Si, extDIA-Ge, extDIA-Si-BN, and extDIA-BN.

a) extDIA

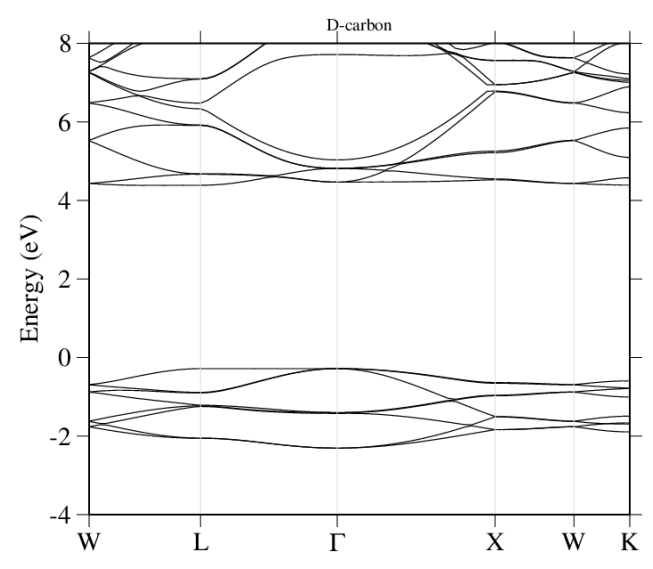

c) extDIA-Ge

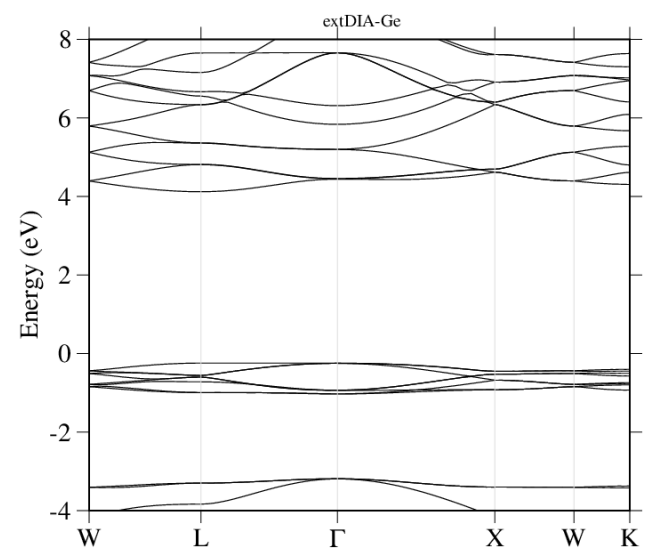

e) extDIA-BN

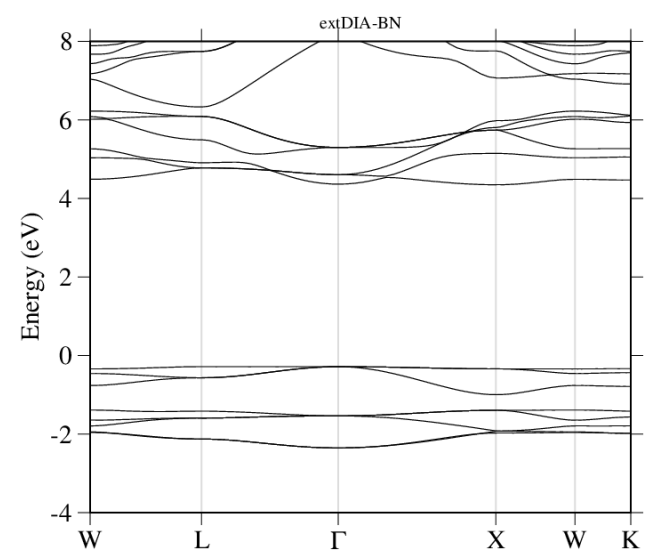

b) extDIA-Si

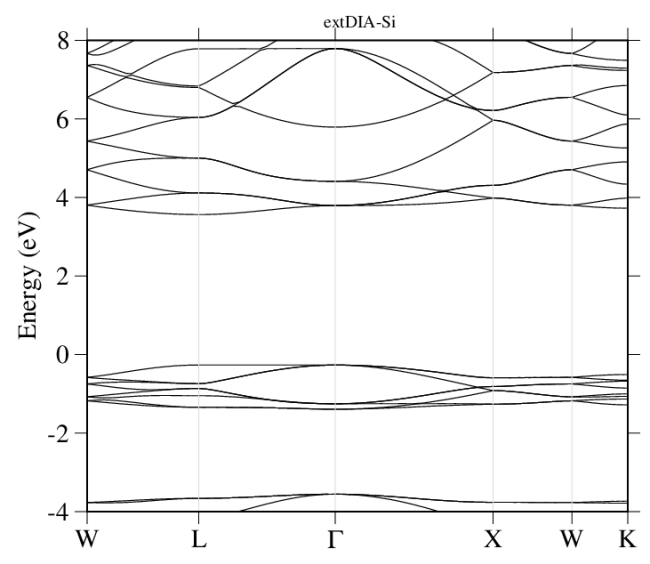

d) extDIA-Si-BN

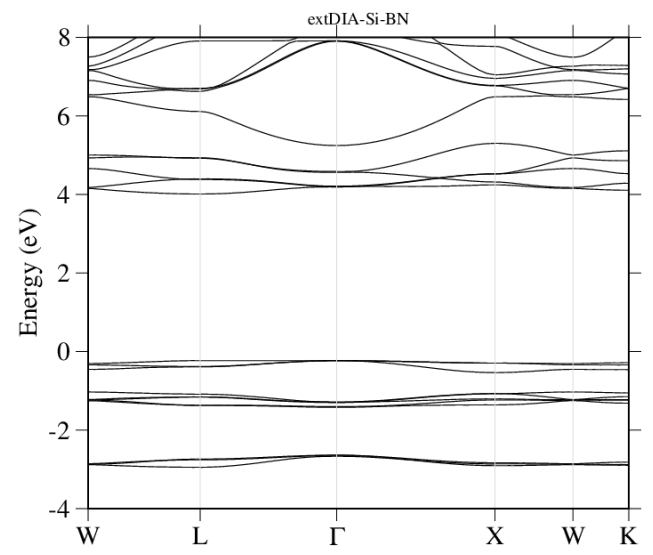




\subsection{Stability of the studied diamond analogues}

The stability of extDIA, extDIA-Si, extDIA-Ge, extDIA-Si-BN and extDIA-BN lattice structures was confirmed by calculating zone center gamma phonons, zone boundary phonons. For this step, we used VASP code with PBE, $400 \mathrm{eV}$ kinetic energy cutoff (except extDIA-Si and extDIA-Si-BN in which $520 \mathrm{eV}$ was used). For zone center phonons, a triclinic primitive unit cell with 10 atoms and $4 \times 4 \times 4$ k-point sampling were used. For zone boundary phonons, $2 \times 2 \times 2$ supercell ( 80 atoms) of the triclinic primitive unit cell and $2 \times 2 \times 2$ k-point sampling were used. We found that both the zone center and the zone boundary phonon frequencies are all real. Three near zero $(<0.2-0.6 \mathrm{~cm}-1)$ frequencies, that corresponds to rigid translational modes and should be zero by symmetry, indicates that our calculations are well converged. Therefore we concluded that all the structures are stable. Full phonon band dispersion along the high symmetry lines in the first Brillouin zone are given in Figure 2S. 
Figure 2S. The phonon band structures of extDIA, extDIA-Si, extDIA-Ge, extDIA-Si-BN, and extDIA-BN. Imaginary frequencies are plotted along the negative frequency axis.

a) extDIA

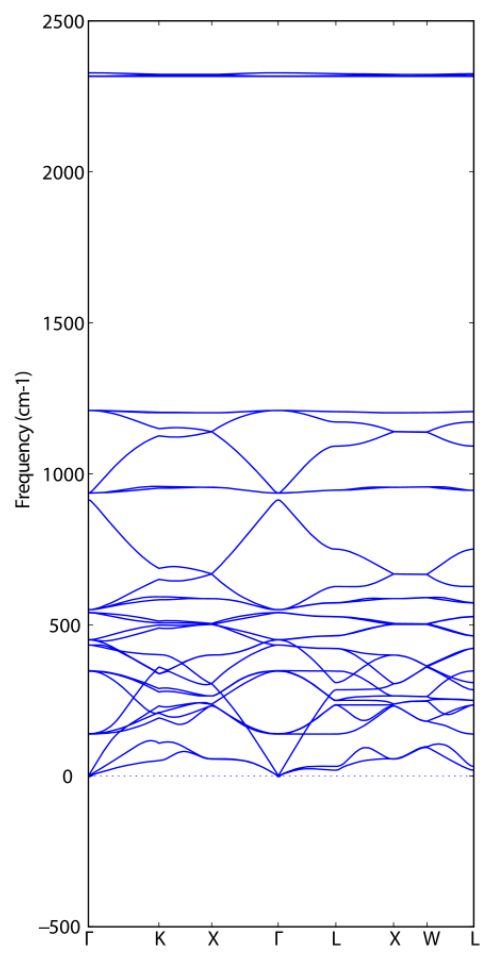

d) extDIA-Si-BN

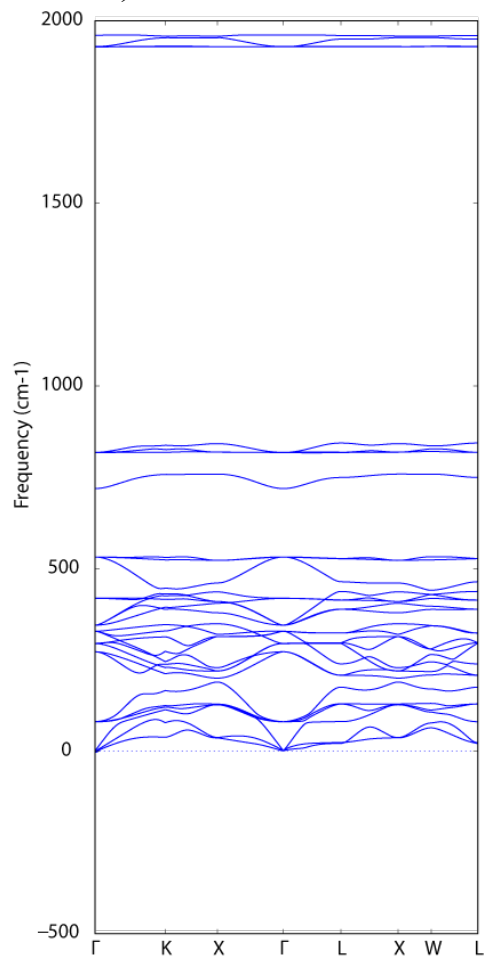

b) extDIA-Si

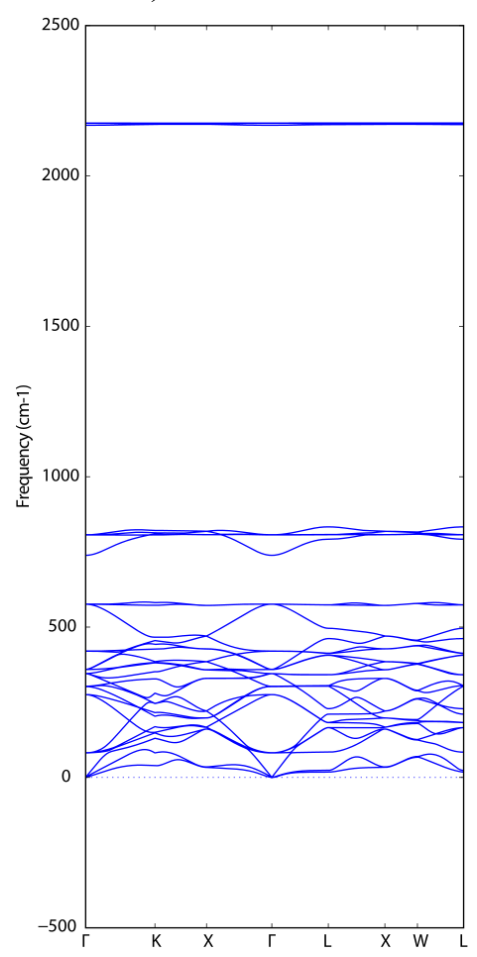

e) extDIA-BN

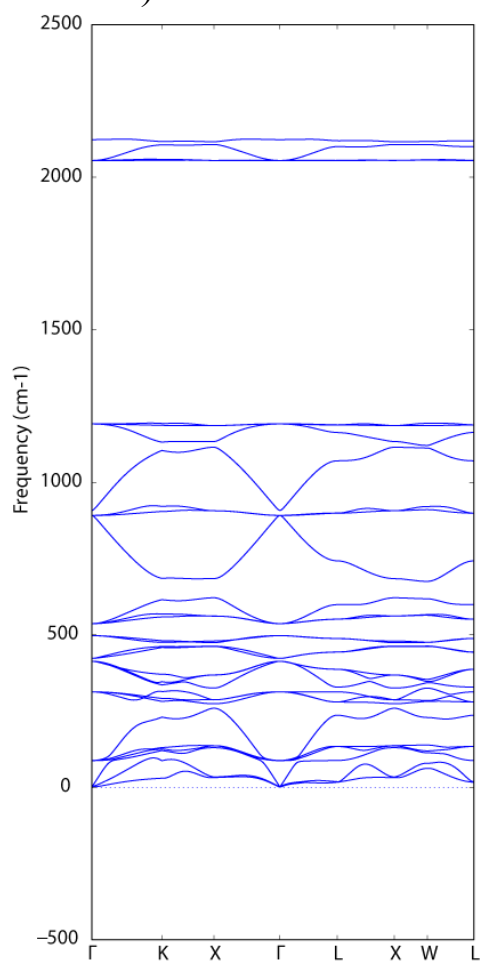

c) extDIA-Ge

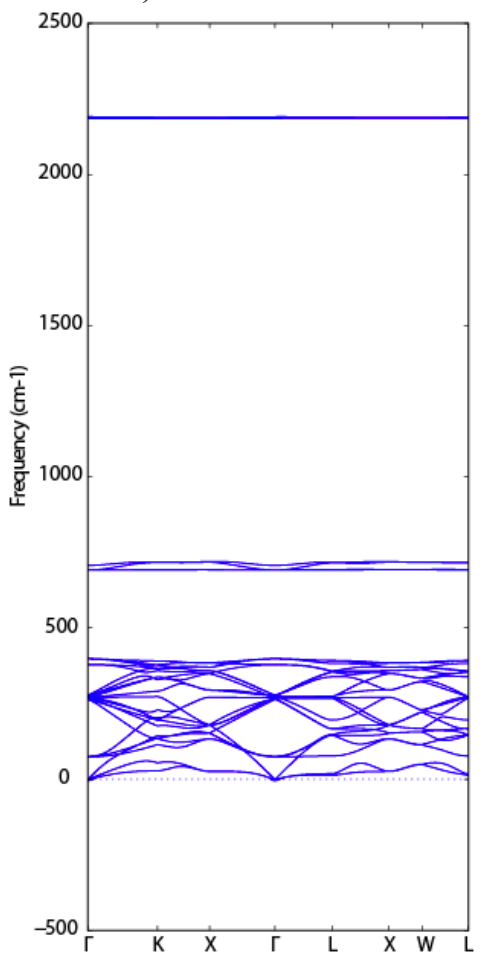




\subsection{Comment on performance of semi-empirical PM6 method}

We would like to comment on the performance of the semi-empirical PM6 method which is a significantly less computationally expensive alternative to DFT, and for which attempts have been made to utilize it for investigation of structures of porous materials (J. Phys. Chem. C, 2013, 117, 12159-12167). The results of PM6 characterization are collected in Table S-1. In general, for most of the considered structures, the PM6 method tends to predict structures with smaller unit cells, smaller pores and higher density. In the case of extDIA, for which we investigated many density functionals, the comparison suggests that PM6 results are within the range predicted by DFT, and are most similar to results with the LDA and PW92 functionals. In the case of other structures, we observe that differences between parameters calculated with DFT-PBE and PM6 are within 2.5\% of each other. A larger difference between DFT-PBE and PM6 is observed for extDIA-Ge, where a much smaller unit cell is predicted with PM6. The unit cell volume reduction by $7 \%$ is also reflected in smaller pore diameters (by up to $5.2 \%$ ) and higher density (7.5\%). The only system for which PM6 predicts a larger unit cell (by $2.5 \%$ ) than DFT-PBE is extDIA-Si-BN. However, both $\mathrm{D}_{\mathrm{i}}$ and $\mathrm{D}_{\mathrm{f}}$ are ca $0.1 \AA$ smaller in the case of the PM6 structures suggesting slight deformation of the unit cell.

Table S-1. Geometrical parameters describing the unit cells of the extended diamond structures and their void space obtained with the semi-empirical PM6 method: the lattice parameters, the unit cell volume (UCV, in $\AA^{3}$ ), helium accessible volume fraction (VF); largest included sphere diameter $\left(D_{i}\right.$, in $\AA$ ); largest free sphere diameter $\left(D_{f}\right.$, in $\AA$ ), and the corresponding materials' crystal density $\left(\mathrm{d}\right.$, in $\left.\mathrm{gcm}^{-3}\right)$. The results obtained with the DFT are also listed to facilitate comparison. The structures were obtained using different DFT exchange-correlation functionals or a semiempirical method as indicated, and the software packages utilized (VASP, Quantum Espresso(QE), Mopac(MPC)) are also indicated after “/” separators.

\begin{tabular}{llllllllllll}
\hline & $\mathbf{a}$ & $\mathbf{b}$ & $\mathbf{c}$ & $\boldsymbol{\alpha}$ & $\boldsymbol{\beta}$ & $\boldsymbol{\gamma}$ & $\mathbf{U C V}$ & $\mathbf{V F}$ & $\mathbf{D i}$ & $\mathbf{D f}$ & $\mathbf{d}$ \\
\hline extDIA/PBE/VASP & 6.806 & 6.764 & 6.819 & 59.780 & 59.535 & 59.931 & 220.68 & 0.100 & 4.42 & 3.46 & 0.9038 \\
extDIA/LDA/VASP & 6.762 & 6.703 & 6.778 & 59.679 & 59.354 & 59.857 & 215.42 & 0.094 & 4.35 & 3.40 & 0.9259 \\
extDIA/PW91/VASP & 6.802 & 6.765 & 6.814 & 59.810 & 59.587 & 59.949 & 220.62 & 0.100 & 4.42 & 3.46 & 0.9040 \\
& & & & & & & & & & & \\
extDIA/PBE/QE & 6.806 & 6.764 & 6.819 & 59.780 & 59.535 & 59.931 & 221.06 & 0.100 & 4.43 & 3.48 & 0.9022 \\
extDIA/LDA/QE & 6.776 & 6.778 & 6.779 & 60.074 & 60.011 & 60.096 & 220.51 & 0.100 & 4.43 & 3.47 & 0.9045 \\
extDIA/PW91/QE & 6.789 & 6.790 & 6.790 & 60.079 & 60.012 & 60.104 & 221.68 & 0.101 & 4.44 & 3.48 & 0.8997 \\
extDIA/PW92/QE & 6.712 & 6.713 & 6.713 & 60.074 & 60.012 & 60.096 & 214.16 & 0.092 & 4.34 & 3.41 & 0.9313 \\
\hline
\end{tabular}




\begin{tabular}{|c|c|c|c|c|c|c|c|c|c|c|c|}
\hline extDIA/PM6 /MPC & 6.797 & 6.712 & 6.839 & 59.364 & 58.979 & 59.364 & 216.80 & 0.095 & 4.35 & 3.38 & 0.9120 \\
\hline extDIA-Si/PBE/QE & 7.932 & 7.944 & 7.936 & 59.938 & 59.946 & 59.961 & 353.13 & 0.226 & 5.53 & 4.61 & 0.7160 \\
\hline extDIA-Si/PM6 /MPC & 7.940 & 7.816 & 7.991 & 60.238 & 59.106 & 60.982 & 351.31 & 0.224 & 5.51 & 4.53 & 0.7197 \\
\hline extDIA-Ge/PBE/QE & 8.185 & 8.152 & 8.208 & 59.946 & 59.597 & 60.236 & 386.60 & 0.255 & 6.02 & 4.83 & 1.0365 \\
\hline $\begin{array}{l}\text { extDIA- } \\
\text { Ge/PM6/MPC }\end{array}$ & 8.035 & 7.942 & 8.080 & 59.466 & 58.770 & 60.037 & 359.75 & 0.234 & 5.76 & 4.58 & 1.114 \\
\hline $\begin{array}{l}\text { extDIA-Si- } \\
\text { BN/PBE/QE }\end{array}$ & 7.996 & 8.068 & 8.077 & 59.418 & 60.331 & 60.362 & 368.69 & 0.170 & 5.58 & 4.07 & 0.7001 \\
\hline $\begin{array}{l}\text { extDIA-Si- } \\
\text { BN/PM6/MPC }\end{array}$ & 7.864 & 8.058 & 8.202 & 58.483 & 62.972 & 62.437 & 376.93 & 0.173 & 5.46 & 4.01 & 0.6848 \\
\hline extDIA-BN/PBE/QE & 6.861 & 6.853 & 6.859 & 60.120 & 59.911 & 60.062 & 228.20 & 0.053 & 4.31 & 2.95 & 0.8972 \\
\hline $\begin{array}{l}\text { extDIA- } \\
\text { BN/PM6/MPC }\end{array}$ & 6.898 & 6.778 & 6.871 & 60.034 & 59.356 & 59.874 & 225.91 & 0.051 & 4.24 & 2.91 & 0.9063 \\
\hline
\end{tabular}

${ }^{a}$ highest symmetry isomer 